\title{
INSTRUMENT FOR MEASURING THERMAL CONDUCTIVITY OF LIQUID AND POWDER
}

\author{
Komal Padwal ${ }^{1}$, Aman Kumar ${ }^{2}$, Akash Babar ${ }^{3}$, Akash Rawat ${ }^{4}$ \\ ${ }^{1}$ Bachelor of Engineering, Mechanical Engineering Department, Trinity Academy of Engineering, Maharashtra, India \\ ${ }^{2}$ Bachelor of Engineering, Mechanical Engineering Department, Trinity Academy of Engineering, Maharashtra, India \\ ${ }^{3}$ Bachelor of Engineering, Mechanical Engineering Department, Trinity Academy of Engineering, Maharashtra, India \\ ${ }^{4}$ Bachelor of Engineering, Mechanical Engineering Department, Trinity Academy of Engineering, Maharashtra, India
}

\begin{abstract}
Determination of thermal conductivity of substance is required in engineering applications. Fourier proposed law for heat conduction that is heat flow rate is directly proportional to area and temperature gradient. Thermal conductivity is ability of material to transfer heat. It is also called as thermal conductivity coefficient. Thermal conductivity varies widely for various engineering materials and it is function of temperature, density and structure. Purpose of this paper is to calculate thermal conductivity of powder, liquid. In this we measured thermal conductivity of plaster of Paris (POP), Water and compared those values with standard values.in this paper we also present variation of temperature with respect to thickness.
\end{abstract}

Keywords: Thermal conductivity, Heat transfer, conduction, Heater, Temperature sensor etc...

\section{INTRODUCTION}

This project main focus will be calculating thermal conductivity of liquid, powder and solid. Heat transfer is very important topic in mechanical engineering that deals with transfer of energy. Physical properties of substances plays important role in design of engineering applications such as heat exchanger.

A literature review shows that many methods are available to determine thermal conductivity based on steady state and transient state. Donald E. Macphee, Seyoon Yoon, Mohammed S. Imbabi presented a paper for estimation of the thermal conductivity of hardened cement paste on the basis of guarded heat flow meter measurements. Thermal conductivity value of cement paste were determined and compared with values reported in literature review[1].Michael Heinrich Rausch, Kamil Krzeminski, Alfred Leipertz, Andreas Paul Froba suggested new guarded plate method to find thermal conductivity of fluids and solid. They worked to minimize heat losses in guarded plate method to increase accuracy of thermal conductivity values [2]. Hosni I. Abu-Mulaweh \& Donald W. Mueller suggested method to find thermal conductivity of liquid and gas [3]. N B Totala1, Pravin Arjun Kasab, Ravi Babasaheb Rathod and Swapnil Sanjay Gunde presented a paper on lagged pipe apparatus to determine thermal conductivity. They presented when temperature increases then thermal conductivity also increases for asbestos powder [4]. Dinesh Kumar, Prakash Chandra worked for development of hot rod method by using water as a coolant medium which can measure heat loss through rod for the steady state measurement of small samples [5].
In this paper authors present a technique that can determine thermal conductivity of solid, liquid, powder by using same instrument. This technique is based on steady state condition. When steady is achieved then readings are taken to calculate thermal conductivity of liquid, powder.

\section{METHODOLOGY}

\section{Liquid / Powder}

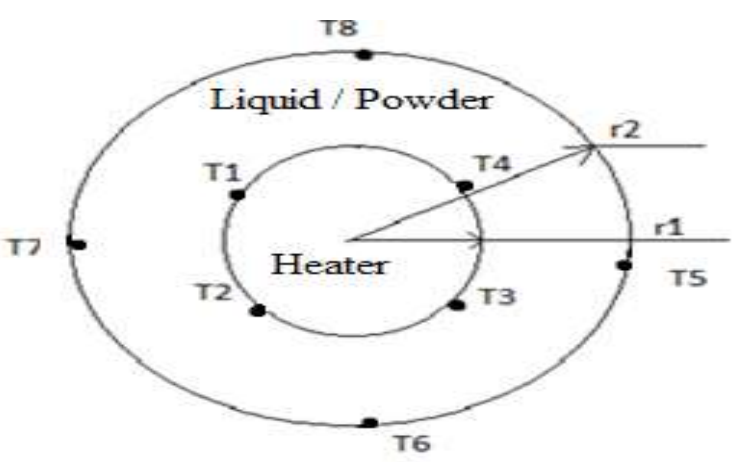

Fig-1:Methodology for determining thermal condctivity of liquid/powder

Experimental setup consist of heater at the center. Heat will transferred only in radial direction. Upper and bottom surfaces of the setup are insulated so to reduce heat losses. When thermal conductivity of liquid or powder is to be determined then powder or liquid placed outside the heater so heat will transferred from heater to liquid or powder. Sensors on surface of heater will measure temperature of heater. Sensors on inside surface of cylinder will measure temperature of liquid or powder. 
According to Fourier's law of conduction,

$$
\begin{aligned}
& \mathrm{Q} \alpha \mathrm{A} \frac{d T}{d r} \\
& \mathrm{Q}=\mathrm{KA} \frac{d T}{d y} \\
& \mathrm{Q}=\mathrm{K}(2 \pi \mathrm{L}) \frac{d T}{d y} \\
& \mathrm{Q} \int_{r 1}^{r 2} \frac{d y}{r}=\mathrm{K}(2 \pi \mathrm{L}) \int_{T i}^{T 0} d T \\
& \mathrm{Q}=\frac{2 \pi L L(T i \bar{i}-T 0)}{\left.\log _{e} \cdot \frac{T^{2}}{T 1}\right)} \\
& \mathrm{Q}=\mathrm{V} * \mathrm{I} \\
& \mathrm{K}=\frac{Q \cdot \log _{\mathrm{s}} \frac{r 2}{r 1}}{2 \pi L(T i-T 0)}
\end{aligned}
$$

Equation (3) is used to calculate thermal conductivity of liquid or powder in this experiment.

$$
\begin{aligned}
& \mathrm{Ti}=(\mathrm{T} 1+\mathrm{T} 2+\mathrm{T} 3+\mathrm{T} 4) / 4 \\
& \mathrm{To}=(\mathrm{T} 5+\mathrm{T} 6+\mathrm{T} 7+\mathrm{T} 8) / 4
\end{aligned}
$$

Where,

$\mathrm{Q}=$ Power input (watt)

$\mathrm{T} 1, \mathrm{~T} 2, \mathrm{~T} 3, \mathrm{~T} 4-\mathrm{Temperature}$ on heater surface

T5, T6, T7, T8- Temperature on inner surface of cylinder $\mathrm{r} 1=$ Radius of heater

r2=Radius of cylinder which contains liquid or powder

$\mathrm{L}=$ length of pipe

$\mathrm{K}=$ Thermal conductivity

\section{DESIGN}

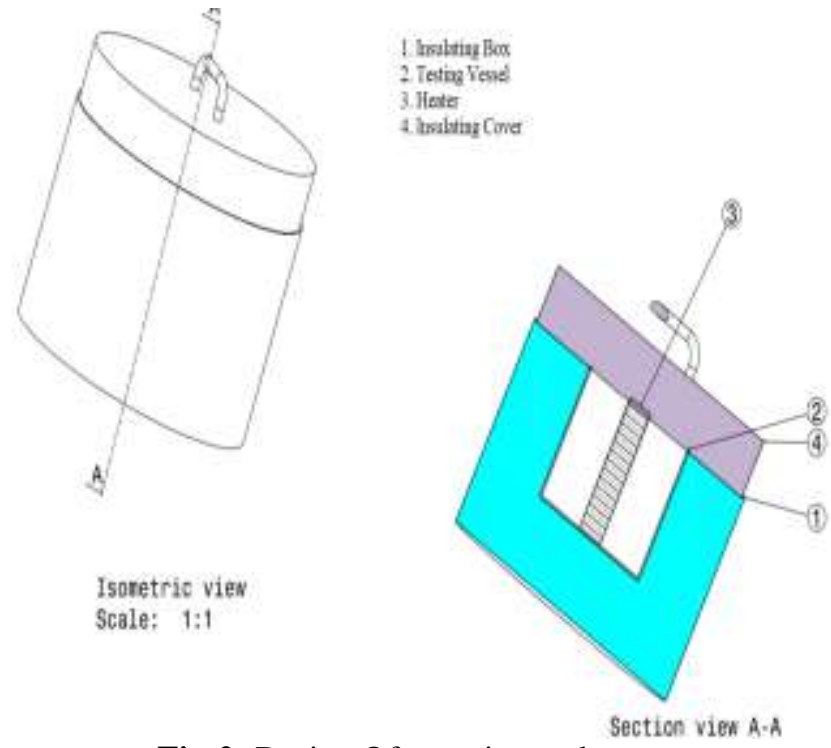

Fig-2: Design Of experimental setup

\section{APPARATUS REQUIRED}

Voltmeter

Ammeter

Temperature indicator

Thermocouple

Heater

\section{PROCEDURE}

1. Start the main switch.

2. By adjusting the dimmer knob give heat input to heater.

3. Allow the unit to reach to the steady state condition.

4. When the steady state is obtained then note down the temperature of sensor.

5. Calculate thermal conductivity by using readings.

\section{RESULT}

\subsection{Plaster of Paris}

Standard thermal conductivity value of Plaster Of Paris is 0.131 . Experimental value of Plaster Of Paris from this setup is 0.1704 for input of 4.9 Watt.

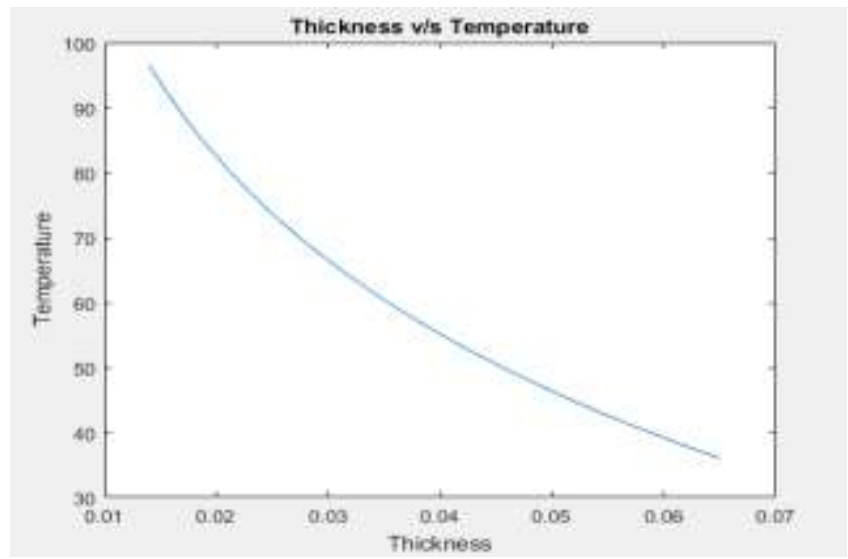

Fig-3: Thickness v/s temperature plot for plaster of Paris

\subsection{Water}

Standard thermal conductivity value of water is 0.59 . Experimental value of thermal conductivity from this setup is 0.6265 for input of 4.96 watt.

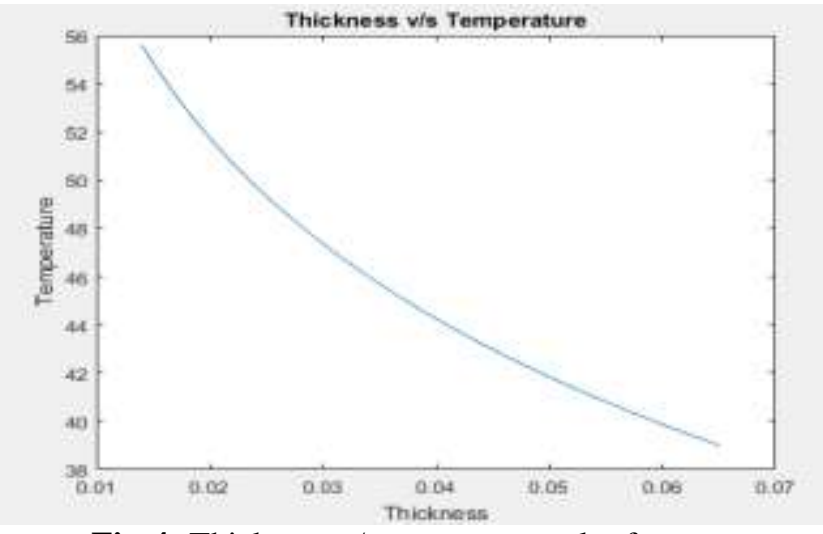

Fig-4: Thickness v/s temperature plot for water 


\section{CONCLUSION}

1. From the plot of thickness v/s temperature of POP and water it is observed that as the thickness increases value of temperature decreases.

2. Value of thermal conductivity of POP that is insulating powder is less than thermal conductivity of water.

\section{REFERENCES}

[1]. Seyoon Yoon, Donald E. Macphee, Mohammed S. Imbabi, "Estimation of the thermal properties of hardened cement paste on the basis of guarded heat flow meter measurements," School of Engineering, Kings College, University of Aberdeen, Aberdeen AB9 2UE, UK.

[2]. Michael Heinrich Rausch, Kamil Krzeminski, Alfred Leipertz , Andreas Paul Fröba, "A new guarded parallel-plate instrument for the measurement of the thermal conductivity of fluids and solids," Universität Erlangen-Nürnberg, PaulGordan-Straße 6, D-91052 Erlangen, Germany.

[3]. Hosni I. Abu-Mulaweh \& Donald W. Mueller, "The determination of the thermal conductivity of fluids," World Transactions on Engineering and Technology Education (C) 2006 UICEE Vol.5, No.3, 2006.

[4]. N B Totala1, Pravin Arjun Kasab, Ravi Babasaheb Rathod and Swapnil Sanjay Gunde, "Thermal conductivity determination by lagged pipe apparatus, 'ISSN 22780149, Vol. 3, No. 2, April 2014 @ 2014 IJMERR.

[5]. Dinesh Kumar, Prakash Chandra, "Development of thermal conductivity measurement test rig for engineering material," e-ISSN: 2278-1684, p-ISSN: 2320-334X, Volume 8, Issue 2 (Jul. - Aug. 2013). 